\title{
Coagulation disturbances in shock
}

\author{
H. G. LASCH \\ Justus Liebig University, Giessen, Germany
}

\begin{abstract}
Summary
Hypocirculation causes hypercoagulability with an increase of Factors V and VIII, and a diminished platelet count. In shock hypercoagulability is followed by hypocoagulability due to loss of Factors I, II, V, VIII and XIII together with thrombocytopaenia and qualitative alterations in platelet properties. This reversal is caused by disseminated intravascular coagulation consuming clotting factors and platelets-consumptive coagulopathy.

Fibrin deposits occur in the peripheral vasculature of the viscera and are associated with a rising oxygen debt, and, in clinical situations, with haemorrhagic diathesis, visceral necrosis and irreversible shock. Localization of the microthrombi is under the influence of catecholamines, ACTH and aldosterone.

Administration of heparin in clinical shock may prevent the development of consumptive coagulopathy, but is without effect if disseminated intravascular coagulation is already present.
\end{abstract}

Anti-fibrinolytic therapy is contraindicated whereas streptokinase-induced fibrinolysis has experimental and clinical justifications.

VESSEL wall and haemostasis are closely interrelated. Experimental and clinical investigations suggest that a continuous physiological turnover of clotting factors and platelets is necessary for the proper functioning of the vessel wall. Intermediary products of intravascular haemostasis such as fibrin monomer and damaged platelets influence permeability, rigidity and resistance of the capillaries. It may very well be possible that insoluble and soluble collagen stemming from the vessel wall form the connection between tissue structure and the blood flowing by the vessel wall.

The central idea of 'latent coagulation' is the continuous breakdown of prothrombin to its subunits, prothrombin, auto-prothrombin $\mathrm{C}$, thrombin, Factor IX and Factor VII, which again determine the global activity of haemostasis in blood. The steady turnover of clotting factors and platelets results in the actual activity of haemostasis. Under normal conditions control mechanisms prevent a manifest clotting of the blood in the circulation. These are:
(1) Inhibitors like anti-thrombokinase, heparin and progressive anti-thrombin, partly released by mast cells, partly by the vessel endothelium, which interrupt momentarily excessive activation processes.

(2) Fibrinolysis which links cellular (leukocytokinase) and plasma factors (anti-thrombin IIIanti-plasmin activity) to haemostasis in the peripheral circulation, and reacts in the same manner as the system of clotting factors.

Under normal conditions, the activation of blood coagulation is automatically followed by the activation of fibrinolysis.

(3) Cellular phagocytosis of the reticulo-endothelial system (RES) plays a decisive role in controlling latent coagulation. It has been shown that the liver and spleen clear activated breakdown substances of haemostasis from the circulating blood. The concentration of Factor VII and Factor IX was found to be higher in the portal vein than in the hepatic veins (Lasch, Mechelke \& Nusser, 1957).

Spaet et al. (1961) have demonstrated that the injection of thromboplastin into the portal vein of rats, that is ahead of the RES, has no bearing on the global activity of haemostasis, whereas the injection of the same amount of thromboplastin into the hepatic vein results in defibrination. By electronmicroscopy, Lee \& McCluskey (1962) have demonstrated material in the Kupffer cells of the liver which resembles fibrin monomer.

Activation of the coagulation system in the periphery and clearance of the intermediary coagulation products thus not only guarantee the latent turnover in the system of haemostasis, but also maintain the eucoagulability of the blood. In this way the circulation itself becomes a decisive factor, since it combines and balances activation, turnover, breakdown and production of the factors of haemostasis. Slowed-down circulation and stagnation of the blood in larger vessels of the blood stream (hypocirculation) result in hypercoagulability. The tendency to develop thrombosis in patients with cardiac insufficiency, post-surgery and post-delivery, can thus be explained.

Alterations in the system of haemostasis are also 
observed in different types of shock. Our experiments in dogs, cats and rabbits have shown that hypercoagulability can be found in the initial stage of haemorrhagic shock, burns, and fat embolism and most pronouncedly in endotoxin shock. In this stage the clotting analysis shows an increased activation of Factor V and Factor VIII and an initial decrease in platelets.

In the later stages of shock the clotting time becomes prolonged. At this point fibrinogen, prothrombin, Factor V, Factor VIII and especially Factor XIII are decreased, and at the same time a decrease in platelets is observed. The maximal amplitude in the thrombelastogram becomes small; $r$ - and $k$-values often become extremely prolonged. Defects in platelet function become obvious: adhesion and agglutination of the platelets are decreased. Analysis of their partial function reveals loss in ATP, serotonin, platelet factor 3 and glycogen. Loss of the platelets in ATP and decrease of the maximal amplitude in the thrombelastogram can directly be correlated.

In summary, it can be said that a stage is reached in the system of haemostasis which corresponds to that after clotting of blood in vitro. Factors and platelets are consumed intravascularly by increased turnover. To define this pathogenetic mechanism in terms of its dynamics we have introduced the term 'Verbrauchskoagulopathie' (consumption coagulopathy).

The quantitative differences in the reaction of haemostasis are dependent on shock and its starting mechanism. The most pronounced changes are seen following endotoxin, fat embolism, burns and haemorrhagic shock, in this order. Endotoxin itself may accelerate the turnover of clotting factors and platelets. Moreover, catecholamines released by the action of endotoxin may effect an increased tendency of the platelets to agglutinate and a loss in platelets in the circulation. In in vitro experiments the addition of catecholamines to platelet-rich plasma results in a pronounced decrease in platelets. Stimulation of the adrenergic system results in the release of free fatty acids into the blood which activate Hageman factor. Crowell \& Smith (1964) attribute an immediate activation of haemostasis to the shifting of the $\mathrm{pH}$ to acidosis. Lipids and intravascular intermediary products of the coagulation, moreover, load the RES. If one considers that stasis of the blood in large areas of impaired microcirculation is followed by a slower flow of blood to the RES, the accumulation of accelerators with consecutive hypercoagulability becomes understandable. At this point intravascular coagulation might start. The breakdown of haemostasis is the consequence of excessive activation in the periphery and overloaded control mechanisms. The diagnosis of intravascular coagulation with consecutive consumption coagulopathy is made in the laboratory.

Fibrin thrombi in the peripheral circulation are the morphologic substrate of a consumption coagulopathy. The extent of fibrin deposition is dependent on the intensity of the intravascular clotting process, the insufficiency of control mechanisms and on special localizing factors. Müller-Berghaus \& McKay (1967) have shown that intravascular coagulation with consecutive fibrin deposition in the renal glomerular capillaries is decreased by pretreatment of the animals with $\alpha$-adrenergic blocking agents such as dibenzamine or dibenzyline. Margaretten and co-workers (1965) have supplied another example for the importance of localization factors: thrombin infusions only result in fibrin deposition and adrenal haemorrhages if ACTH is administered together with the thrombin infusion. McKay \& Müller-Berghaus (1968) have demonstrated that an activation of Hageman factor by ellagic acid with simultaneous inhibition of fibrinolysis by $\varepsilon$-aminocaproic acid only results in fibrin deposition if noradrenaline is administered at the same time.

In the same manner morphologic equivalents can be shown in patients who have died from shock with consumption coagulopathy. Clinical and laboratory data may possibly correspond to the morphologie substrate if the autopsy is performed immediatel after death and post mortem lysis is prevented Sandritter \& Lasch (1967) were able to demonstrate fibrin depositions in various organs in about $70 \%$ of patients who had died in shock.

The consequences of consumption coagulopathy which manifest themselves in acute circulatory failure are (Table 1):

(1) A haemorrhagic diathesis which is not obligatory and which is caused by the breakdown of haemostasis. Suggilation and petechiae are observed. Secondary fibrinolysis can enhance the haemorrhagic diathesis by generation of fibrinogen and fibrin split products. Split products may interfere with the structure of a haemostatic plug. In addition to this, split products inhibit the aggregation of platelets. Laboratory analysis should differentiate between bleeding caused by consumption coagulopathy and bleeding caused by hyperfibrinolysis.

(2) Disturbances in the blood flow of various organs with secondary damage: renal cortical necrosis and acute renal failure, adrenal haemorrhage and meningococcal sepsis (Waterhouse-Friedrichsen), pituitary necrosis with global insufficiency (Sheehan), pulmonary microthrombo-embolism with pulmonary hypertension and acute cor pulmonale, thrombosis of the hepatic veins (Budd-Chiari syndrome), portal hypertension and ascites. These organic lesions may 
TABLE 1. Diagnosis of intravascular coagulation and fibrinolysis

Consequences of consumption coagulopathy

\begin{tabular}{ll}
\hline 1. Haemorrhagic diathesis & $\begin{array}{l}\text { (a) Caused by consumption of coagulation factors } \\
\text { and thrombocytes. Intravenous coagulation }\end{array}$ \\
(b) Caused by compensatory fibrinolysis and hyper- \\
fibrinolysis \\
$\begin{array}{l}\text { 2. Local impairment of microcirculation } \\
\text { followed by necrosis }\end{array}$ & $\begin{array}{l}\text { Bileral cortical necrosis of the kidney, acute renal } \\
\text { failure } \\
\text { Necrosis of the hypophysis, Sheehan's syndrome } \\
\text { Necrosis of the adrenal cortex, Waterhouse- } \\
\text { Friedrichsen syndrome } \\
\text { Pulmonary hypertension }\end{array}$ \\
$\begin{array}{l}\text { 3reversible shock } \\
\text { Generalized impairment of }\end{array}$ &
\end{tabular}

determine the course of the disease and the life of the patient at a time when the initial circulatory failure has already been overcome.

(3) The protraction of circulatory insufficiency caused by an increasing oxygen debt of heart and vessels, so called irreversible shock.

Generalized intravascular clotting occurring in the course of shock probably enhances existing disturbances of the microcirculation. Experimental and clinical investigations have shown an increasing oxygen debt in the tissue with the beginning of consumption coagulopathy. Registration of oxygen consumption in endotoxin shock reveals an increasing oxygen debt in the tissue even before the decrease in blood pressure, circulation time and arterial oxygen saturation. At values above $140 \mathrm{ml} / \mathrm{O}_{2} / \mathrm{kg}$ body weight tissue shock becomes irreversible. Metabolites from hypoxic tissue, acidosis, stasis and intravascular clotting with fibrin and platelet thrombi form a vicious circle. Metabolites may act as vasodilators by inhibiting the reaction of precapillary vessels to neural and hormonal stimulation (Shoemaker, 1967). Microscopic examination of the heart shows fibrin deposits in the microcirculation of coronary arteries. This is in accordance with the findings of Crowell \& Smith (1964) who postulate the increasing oxygen debt of the heart as an essential cause for the irreversibility of shock (acute cardiac insufficiency). In the same way organs with impaired microcirculation like the liver and kidney will influence the circulatory situation.

The question now arises whether changes in the system of haemostasis are only epiphenomena of shock or whether they become important in the pathogenesis of circulatory failure itself. Hardaway (1966) has demonstrated that by improving the circulatory situation in haemorrhagic shock by means of volume substitution and blockade of the $\alpha$-adrenergic system, laboratory data for intravascular clotting improved as well. This is not sufficient evidence of its pathogenesis. Further study has to be devoted to controlling shock by successfully influencing haemostasis.
Anticoagulants like heparin which are only administered after the onset of shock may interrupt consumption coagulopathy, but do not have any effect whatsoever on the course of shock. This is not true for its prophylaxis. Rabbit experiments show that a deadly oxygen debt is prevented by continuous heparin administration if an otherwise lethal dose of endotoxin is administered. This experimental finding is in accordance with clinical data obtained at the Women's Hospital in Heidelberg (Kuhn \& Graeff, 1968) where in the last 3 years no single death was observed in patients suffering from febrile septic abortions who had been prophylactically treated with heparin. Consequently, the next step should be to test therapeutic fibrinolysis in patients with shock. Animal experiments conducted on the successful administration of fibrinolysins in haemorrhagic shock in the dog (Hardaway \& Burns, 1963), the cat (Lasch et al., 1961) and the rabbit are available. It was shown that the survival rate of dogs in haemorrhagic shock can be improved from 8 to $58 \%$ by treatment with streptokinase. Rabbit experiments demonstrate that the lethal oxygen debt in endotoxin shock can still be compensated if at the right time fibrinolysis is induced by infusion of streptokinase.

The treatment of shock in humans by means of fibrinolysis therapy with streptokinase has yielded promising results in some cases. Recently, we have measured oxygen consumption and oxygen debt in shock patients with the method developed by Crowell \& Smith (1964). Cases of patients in cardiogenic shock after cardiac infarction show that the incurred, registered oxygen debt can be compensated by streptokinase therapy. These results are no final proof; they indicate, however, that changes in the haemostatic mechanism are not epiphenomena of shock but important pathogenetic factors in its course especially in its critical irreversible stage.

New aspects arise as a practical consequence of this interrelation between haemostasis and circulation in shock. In order to be able to judge the course of shock, data from the coagulation laboratory in 
addition to all other laboratory data should be available. Hypercoagulability, intravascular clotting and consumption coagulopathy are criteria of the humoral participation of the blood in acute circulatory failure. To give these criteria their proper role in pathophysiology and diagnosis might provide new opportunities for the therapy of shock.

\section{References}

Crowell, J.W. \& SMith, E.E. (1964) Oxygen deficit and irreversible hemorrhagic shock. Amer. J. Physiol. 206, 313.

HaRdaway, R.M. (1966) Syndromes of Disseminated Intravascular Coagulation with special Reference to Shock and Hemorrhage. Thomas, Springfield, Illinois.

HARDAWAY, R.M. \& BURNS, I.W. (1963) Mechanism of action of fibrinolysin in the prevention of irreversible hemorrhagic shock. Ann. Surg. 157, 305.

Kunn, W. \& Graeff, H. (1968) Prophylaktische Massnahmen bei septischem Abort. Septischer Abort und bakterieller Schock. Springer, Berlin.

LasCh, H.G., Mechelke, E. \& Nusser, E. (1957) Uber Anderungen der Gerinnungsfaktoren Prothrombin und
VII bei hyper- und hypozirkulatorischen Kreislaufumstellungen der Katze. Dtsch. Arch. klin. Med. 204, 1.

LASCh, H.G., Mechelke, K., Nusser, E. \& DaOud, F. (1961) Der Einfluss der Fibrinolyse auf den Verlauf des haemorrhagischen Schocks. Klin. Wschr. 39, 1137.

LeE, L. \& MCCluskey, R.T. (1962) Immunohistochemical ज़ demonstration of the reticulo-endothelial clearance of circulating fibrin aggregates. J. exp. Med. 116, 611.

Margaretten, W., Elting, J., Rothenberg, J. \& McKay, D.G. (1965) Experimental adrenal hemorrhage in the generalized Shwartzman reaction. Lab. Invest. 14, 687.

McKay, D.G. \& Müller-Berghaus, G. (1968) Hagemannfactor (HF) and the generalized Shwartzman reaction (GSR). Fed. Proc. 27, 436.

Müller-Berghaus, G. \& MCKay, D.G. (1967) Prevention of the generalised Shwartzman reaction in pregnant rats by $\alpha$-adrenergic blocking agents. Lab. Invest. 17, 276.

SANDRITTER, W. \& LASCH, H.G. (1967) Pathologic aspects of shock. Meth. Achievm. exp. Path. 3, 86.

Shoemaker, W.C. (1967) Shock: Chemistry, Physiology and Therapy. Newton Kugelmass, New York.

Spaet, T.H., Horowitz, H.L., Zucker-Franklin, D., ơ Cintron, J. \& Biezenski, J.J. (1961) Reticuloendothelial N clearance of blood thromboplastin by rats. Blood, 17, 196. 